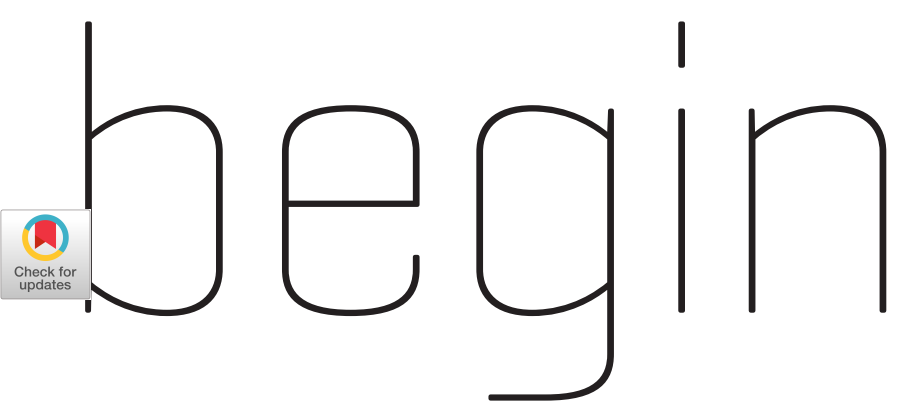

\title{
You Mess The Kitchen, You Do The Dishes
}

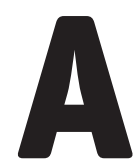

s 2020 nears its end, we reflect on a year marked by worldwide disruption.

Distance was no longer an excuse to miss a meeting. Students and teachers alike were still adapting to online learning, to varying success. From Bangkok to Beirut, Santiago to Suez, Minsk to Minneapolis, picket fences became picket signs as dissenters donned masks and took a stand in the streets for reform, rights, and respect. Plans, jobs, and lives were lost as a pandemic severed any semblance of normalcy-and speaking of COVID, is that still going around? All the while, fires raged across the Australian Outback and the Amazon, locusts swarmed Africa and Asia, and only the Arctic worked up more of a sweat than nurses on the frontlines.

During extraordinary times, we find comfort in signs of normalcy: outdoor dining, hundreds of Among Us matches, reuniting with old friends and our favorite sitcom casts, longing for old ways to return. But make no mistake, we are living in a transition-and a shattering of norms. Some norm destruction is welcome: perhaps attending work and conferences from home is easier for many families and international teams-and better for the environment. More unsettling, we're witnessing examples-from Hong Kong to the United States-of how fragile the foundations and norms of democracy can be.

Technology plays an increasingly outsized role in society. This year only solidified tech's reach. Glued to screens at home, the internet became our primary window to the world, providing a platform for activists to reflect on society's systemic ills, as well as to conspiracy theories undermining confidence in science and democracy. Governments recognized the importance of universal access to the internet-and sought to surveil it and control its discourse. Meanwhile, biased facial recognition and personal data crunching-sometimes

with help from private industry-are continually used to deploy algorithms for policing and perfor-

\section{How can you tell when you're on the cusp of transition?"}

mance evaluation that are difficult to keep in check. While tech powers change, humanity ultimately got itself into this mess. Now, it's our job to clean things up.

In "Agents of Change," we feature 11 agents and collective movements working across the globe to build a habitable, equitable future empowering individual agency; the government agents who frequently stand in the way; and the technology that drives it all. Their stories demonstrate the power of research and activism in shaping our changing world, and we hope they'll convince you to connect your own work to addressing society's greatest challenges.

Our first set of agents share stories at key intersections of tech and public policy: censorship, surveillance, and data privacy. Leading the charge this year is Glacier Kwong, Hong Kong activist and law Ph.D. student at the University of Hamburg, Germany. Kwong describes the radical increase in surveillance and seizures in her home from a National Security Law enacted earlier this year, and advocates for secure and private tech that keeps her fight alive.

Complex battles between privacy laws across borders can ultimately benefit both sides. Austrian activist Maximilian Schrems twice upended decades of data privacy compromises between the European Union and the United States, and put America's surveillance in check. Mihir Kshirsagar of Princeton details how Schrems turned a class assignment into a seven-year legal battle, motivated by Edward Snowden's U.S. surveillance leaks, that ultimately upheld privacy rights for European citizens, and sent authorities on both coasts scrambling for a fix.

With too many data collectors to keep track of, even stringent privacy laws may not be enough to ensure data sharing is an individual choice. By taking collective action, however, we can force changes to collectors themselves. Jonathan Zong, Ph.D. student at MIT, discusses how society can collectively refuse pervasive data collection by industry, and how everyone from rising tech researchers and activists to average users can play a role.

When governments censor network traffic, users turn to evasion tools. Kevin Bock and Professor Dave Levin from the University of Maryland describe Geneva, their automated censorship evasion tool leveraging genetic algorithms 


\section{0 of organizations will shift from piloting to operationalizing Al by the end of 2024, driving a five times increase in streaming data and analytics infrastructures.}

to find bugs in censorship systems faster than regimes can patch them. Geneva's revolutionary "server-side evasion" technique, once thought impossible, allows services to provide censorship evasion without any user action.

The next agents investigate how tech reinforces societal biases and power relationships harming marginalized groups, and how examining our own biases can (sometimes) turn tech around to improve equity. Shivangi Narayan spent her Ph.D. at Jawaharlal Nehru University exploring New Delhi's algorithmic policing system, and argues for its dismantling. Narayan describes how the roots of India's caste system are ever-present in myriad ways biasing the algorithm against poor communities and how humans play a vital role in the system's feedback loop of oppression.

Addressing systemic bias in tech requires examining bias embedded therein. Stephanie T. Jones and Natalie Melo, Ph.D. students at Northwestern University, discuss systems of power, specifically anti-Blackness, coded into the technology we learn about and use everyday, and how computer science education is not devoid of political meaning. They suggest questions for both researchers and educators to examine how their work addresses these systems of power rather than accepting them as a part of the CS story.
Those controlling the creation and interpretation of data can distort and influence reality, from reshaping basic geography to stifling lived experiences of marginalized students. Benjamin Xie, Ph.D. candidate at the University of Washington, explores overlooked factors behind student performance in large CS programs, and how detached interpretations of data can lack the context necessary to make meaningful improvements for marginalized students. Xie discusses his work at Code.org using data science as a tool amplifying and integrating marginalized voices into curricula that equitably serves students with diverse backgrounds and challenges.

Equitable education requires every student to participate in the conversation, and COVID lockdowns have exposed the digital divide to public attention. Andre van Zyl and Professor David Johnson of the University of Cape Town, South Africa, describe iNethi, a community-driven WiFi mesh network combined with locally-hosted cloud services connecting a lowincome peri-urban township in Cape Town. Van Zyl and Johnson provide datadriven insights about what it takes to run a reliable network in a scarce environment and how they adapted iNethi to provide free COVID-19 and classroom resources.

Our next agents are leveraging environmental data to save the planet that makes everything possible. Priya Donti, Ph.D. student at Carnegie Mellon, tells her story finding her place within the group of thinkers fighting climate change. Donti describes her journey interviewing over 150 people worldwide about next-generation energy systems, and she shares lessons learned from building Climate Change AI, an organization she co-founded to galvanize researchers and professionals to fight climate change using machine learning.

The first step in utilizing environmental data is efficient and sustainable collection. Marine research is particularly in need of smarter devices to collect ocean data. Sayed Saad Afzal, Ph.D. student at MIT, dives into his research on networked underwater sensors. Afzal illustrates the challenges he and his colleagues tackled to build the first-ever underwater "backscatter" sensor network: no batteries required.

These "Agents of Change" have dedicated significant portions of their lives to their ideas and movements. We hope they inspire you to find your own path. But before you settle on your life's mission, there's a small action that many of us can take to drive great change: vote! We conclude this XRDS issue with insights about the future of voting tech from Dr. Matt Bernhard, research engineer at VotingWorks and recent $\mathrm{Ph}$. D. graduate of the University of Michigan. Hint: the revolution might be postmarked.

We leave you with a thought exercise. One of our fondest discoveries of quarantine free time has been the wealth of primordial video cameras in the 1890 s (watch a few yourself!). It is fascinating to see dapper gents and factory workers alike staring in bemusement, for a few moments, at the unknown contraption before them. Little did they know the future would be staring back at them almost 130 years later. How can you tell when you're on the cusp of transition? What change could they not foresee? What aspects of our lives will seem completely foreign in 100, even 10 years? Who will be studying us with the same fascination? And most importantly, what change will you be a part of?

Thanks to $X R D S$ for thoroughly supporting this issue, and to our phenomenal authors for sharing their stories, insights, and visions for the future. We are grateful for the enthusiasm and energy that each author contributed to make this issue possible. Please reach out to authors to learn more about their efforts.

See you all in 2021! It's just a matter of time.

—Ross Teixeira, Guest Editor

Henri Maxime Demoulin, Lead Editor

DOI: 10.1145/3439728 Copyright held by author. 